\title{
Utility of Direct Peroral Cholangioscopy Using a Multibending Ultraslim Endoscope for Difficult Common Bile Duct Stones
}

\author{
Won Myung Lee ${ }^{1}$, Jong Ho Moon ${ }^{1}$, Yun Nah Lee ${ }^{1}$ II Sang Shin ${ }^{1}$, Tae Hoon Lee ${ }^{2}$, Jae Kook Yang ${ }^{2}$, Sang-Woo \\ $\mathrm{Cha}^{3}$, Young Deok $\mathrm{Cho}^{3}$, and Sang-Heum Park ${ }^{2}$ \\ ${ }^{1}$ Digestive Disease Center and Research Institute, Department of Internal Medicine, Soonchunhyang University Bucheon Hospital, \\ Soonchunhyang University School of Medicine, Bucheon, ${ }^{2}$ Digestive Disease Center and Research Institute, Department of Internal \\ Medicine, Soonchunhyang University Cheonan Hospital, Soonchunhyang University School of Medicine, Cheonan, and ${ }^{3}$ Digestive \\ Disease Center and Research Institute, Department of Internal Medicine, Soonchunhyang University Seoul Hospital, Soonchunhyang \\ University School of Medicine, Seoul, Korea
}

See editorial on page 499.

\section{Article Info}

Received August 4, 2021

Revised September 13, 2021

Accepted September 24, 2021

Published online January 7, 2022

\section{Corresponding Author}

Jong Ho Moon

ORCID https://orcid.org/0000-0002-3946-9944

E-mail jhmoon@schmc.ac.kr
Background/Aims: Treatment options for difficult bile duct stones are limited. Direct peroral cholangioscopy (POC)-guided lithotripsy may be an option. A newly developed multibending (MB) ultraslim endoscope has several structural features optimized for direct POC. We evaluated the utility of direct POC using an MB ultraslim endoscope for lithotripsy in patients with difficult bile duct stones.

Methods: Twenty patients with difficult bile duct stones, in whom stone removal using conventional endoscopic methods, including mechanical lithotripsy, had failed were enrolled from March 2018 to August 2019. Direct POC-guided lithotripsy was performed by electrohydraulic lithotripsy or laser lithotripsy. The primary outcome was complete ductal clearance, defined as the retrieval of all bile duct stones after lithotripsy confirmed by balloon-occluded cholangiography and/or direct POC.

Results: The technical success rate of direct POC was $100 \%(20 / 20)$, and the free-hand insertion rate was 95\% (19/20). Direct POC-guided lithotripsy, attempted by electrohydraulic lithotripsy in nine patients (45\%) and laser lithotripsy in 11 patients (55\%), was successful in 95\% (19/20) of the patients. Complete ductal clearance after direct POC-guided lithotripsy was achieved in $95 \%$ (19/20) of patients. Patients required a median of 2 (range, 1-3) endoscopic retrograde cholangiopancreatography sessions for complete stone removal. Adverse event was observed in one patient $(5 \%)$ with hemobilia and was treated conservatively.

Conclusions: Direct POC using an MB ultraslim endoscope was safe and effective for lithotripsy in patients with difficult bile duct stones. (Gut Liver 2022;16:599-605)

Key Words: Peroral cholangioscopy; Common bile duct; Endoscopes; Gallstones

\section{INTRODUCTION}

Approximately $10 \%$ to $15 \%$ of patients with bile duct stones experience treatment failure using conventional endoscopic stone removal with mechanical lithotripsy (ML). ${ }^{1,2}$ Additional lithotripsy procedures, such as electrohydraulic lithotripsy (EHL) and laser lithotripsy (LL), can be useful for treating difficult bile duct stones. Cholangioscopic guidance during such procedures may be pivotal for safety and effectiveness.
Peroral cholangioscopy (POC) has been used for direct visualization of the bile duct with simultaneous therapeutic intervention. For this purpose, direct POC using an ultraslim upper endoscope has been proposed. ${ }^{3,4}$ High image quality, a wide range of procedures because of the large (2 $\mathrm{mm}$ ) working channel, and reusability are advantages of direct POC using an ultraslim upper endoscope. However, technical difficulty and positional instability of the endoscope after removal of accessories are drawbacks. ${ }^{3}$

A multibending $(\mathrm{MB})$ ultraslim endoscope has been 
developed as a dedicated cholangioscope for direct POC. It has two bending channels for improved angulation toward the major papilla and two working channels for simultaneous procedures.

We evaluated the utility of direct POC for lithotripsy in patients with difficult bile duct stones using an MB ultraslim endoscope.

\section{MATERIALS AND METHODS}

\section{Patients and study design}

We reviewed prospectively collected and maintained data from March 2018 to August 2019 at a single tertiary referral center. The inclusion criteria were patients with bile duct stones in whom conventional endoscope stone removal, including ML, had failed; previous sphincteroplasty such as endoscopic sphincterotomy and/or endoscopic papillary balloon dilation; and distal common bile duct (CBD) dilation $\geq 8 \mathrm{~mm}$. The exclusion criteria were stricture of the distal CBD, diagnosis of periampullary cancer, presence of intrahepatic duct stones, bleeding tendency (platelet count $<60,000 / \mathrm{mm}^{3}$ or international normalized ratio $>1.5$ ), and refusal to participate.

Informed consent was obtained from all participants. The study protocol was registered at the UMIN Clinical Trials Registry (registration number: UMIN000025048). The study protocol was approved by the Institutional Review Board of Soonchunhyang University Bucheon Hospital (IRB number: SCHBC 2014-10-020).

\section{Devices}

The third-generation MB ultraslim endoscope (CHFY0010; Olympus Medical Systems, Co., Ltd., Tokyo, Japan) is a forward-viewing device with a working length of 1,330 $\mathrm{mm}$. The outer diameter of the endoscope is $4.9 \mathrm{~mm}$ at the distal end and $7.0 \mathrm{~mm}$ at the insertion tube. The endoscope has two bending sections, for two-way deflection in a single plane. It provides angulation of $200^{\circ}$ upward and $100^{\circ}$ downward in the distal section, and $90^{\circ}$ upward and downward in the proximal section. The endoscope is equipped with two working channels, 2.2 and $1.0 \mathrm{~mm}$ in diameter. ${ }^{5}$

\section{Procedures}

All patients were placed in the prone position under intravenous sedation induced by midazolam and meperidine. Prior to the procedure, prophylactic systemic antibiotics were administered. The MB ultraslim endoscope was inserted and advanced into the duodenal bulb. For insertion into the distal CBD, the second bending part of the endoscope was positioned at an acute angle. The endoscope was advanced directly into the bile duct through the major papilla, and cholangioscopic examination and therapeutic interventions were performed.

Insertions were primarily intended to be free-hand. If free-hand insertion was not possible, however, intraductal balloon insertion was done using a modified 5-F balloon catheter for direct POC (Endoskopie; MTW, Wesel, Germany).

The modified 5-F balloon catheter for direct POC is 5-F in diameter and $240 \mathrm{~cm}$ in length with a maximum balloon diameter of $12 \mathrm{~mm}$. It is designed to remain inflated during replacement of the duodenoscope with an ultraslim endoscope. ${ }^{6}$ For lithotripsy procedures, an electrohydraulic shockwave generator (Lithotron EL-27; Olympus Optical, Hamburg, Germany) was used for EHL (Figs 1, 2 and Supplementary Videos 1, 2). The Holmium laser system (Lumenis, Chicago, IL, USA) or FREDDY laser system (Freddy U100Plus Lithotripsy Laser; World of Medicine,
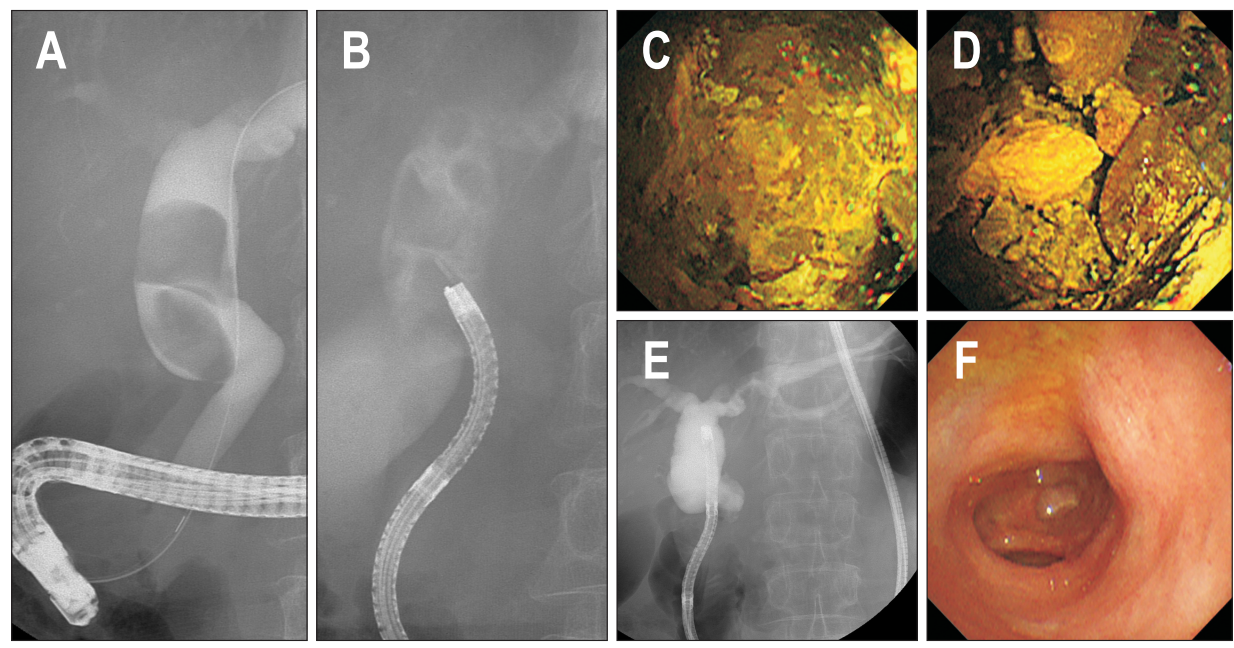

Fig. 1. Direct peroral cholangioscopy-guided electrohydraulic lithotripsy (EHL) using a multibending ultraslim endoscope for retained common bile duct stones. (A) Retained common bile duct stones. EHL for retained common bile duct stones. Fluoroscopic view (B) and cholangioscopic view (C). (D) Fragmented stone after EHL. Confirmation of ductal clearance by fluoroscopy (E) and cholangioscopy (F). 

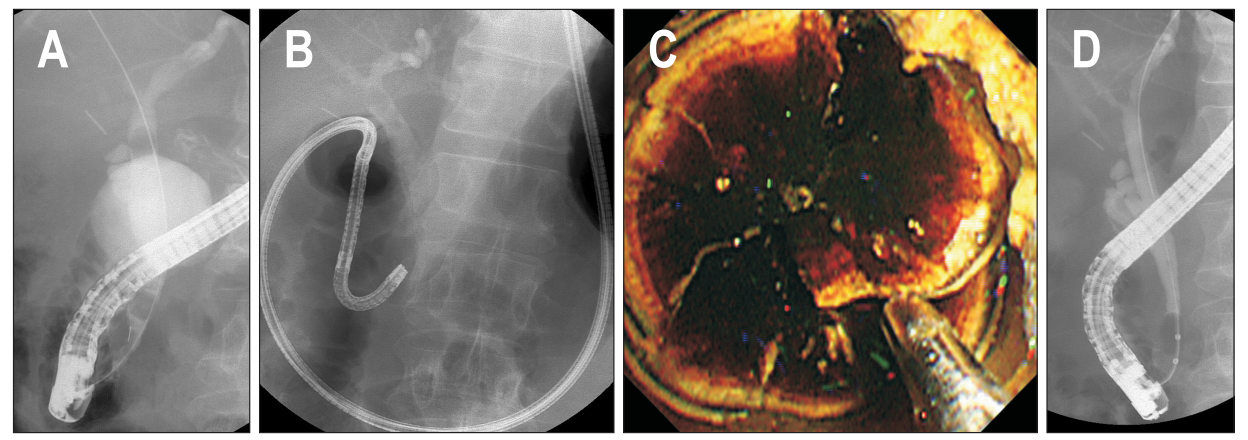

Fig. 2. Direct peroral cholangioscopy (POC)-guided electrohydraulic lithotripsy (EHL) using a multibending ultraslim endoscope for an impacted distal common bile duct stone. (A) Cholangiogram showing an impacted distal common bile duct stone. (B) Direct POC with a multibending ultraslim endoscope for EHL. (C) Cholangioscopic view of the impacted stone and EHL under direct visualization. (D) Confirmation of ductal clearance by occluded cholangiogram.
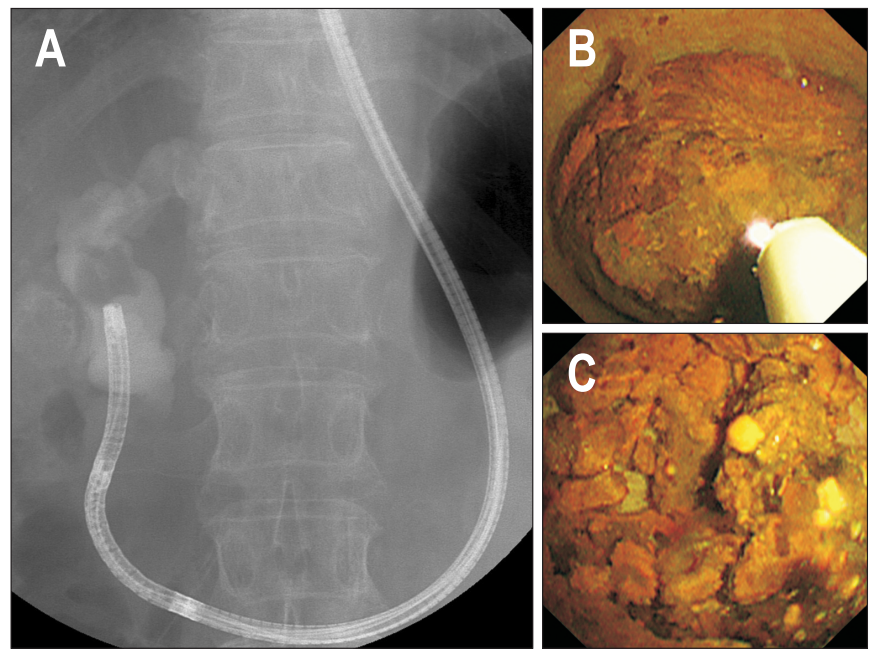

Ludwigsstadt, Germany) was used for LL (Fig. 3). For removing stone fragments, a standard therapeutic duodenoscope (TJF-260V; Olympus, Tokyo, Japan) was used. If needed, mechanical lithotripters (BML 3Q, Olympus; or Trapezoid RX Wireguided Retrieval Basket, Boston Scientific, Marlborough, MA, USA) were applied to facilitate stone removal. Bile duct clearance was confirmed by balloon-occluded cholangiography or direct POC in patients who underwent lithotripsy.

\section{Definitions and outcome measurements}

The primary outcome was complete ductal clearance. Bile duct clearance was defined as retrieval of all bile duct stones after lithotripsy by direct POC. The secondary outcomes were technical success of direct POC, technical success of direct POC-guided lithotripsy, rate of additional ML use, number of endoscopic retrograde cholangiopancreatography (ERCP) sessions for complete stone removal, and procedure time for direct POC-guided lithotripsy.

Technical success of direct POC was defined as suc-

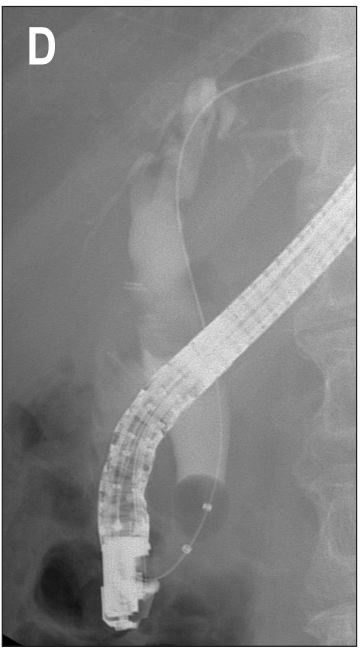

Fig. 3. Direct peroral cholangioscopy-guided laser lithotripsy (LL) using a multibending ultraslim endoscope for a retained common bile duct stone. (A) Cholangiogram of a retained stone in the common bile duct. (B) Cholangioscopic view of LL. (C) Fragmented stone after LL. (D) Confirmation of ductal clearance by occluded cholangiogram.

cessful advancement of the endoscope up to the stone in the biliary tree within 15 minutes, via free-hand insertion or with intraductal balloon assistance. Technical success of direct POC-guided lithotripsy was defined as advancement of the endoscope into the bile duct and subsequent successful initiation of lithotripsy. The procedure time for direct POC-guided lithotripsy was the time between entry into the pylorus by the $\mathrm{MB}$ endoscope and the end of bile duct stone lithotripsy. A single session of endoscopic stone removal was up to 45 minutes in duration.

\section{Adverse events}

Adverse events related to endoscopy, such as cholangitis, perforation, pancreatitis, hemobilia, and air embolism were recorded in all patients for 7 days post-procedure. Adverse events were defined according to the American Society for Gastrointestinal Endoscopy criteria. ${ }^{7}$ 


\section{RESULTS}

During the study period, 448 patients visited our institution for CBD stones. Among them, 39 patients in whom treatment of bile duct stones by conventional endoscopic methods had failed were screened for study entry; 19 of those patients were excluded due to CBD dilation $<8 \mathrm{~mm}$ $(n=16)$ or refusal to participate $(n=3)$. Finally, 20 patients who met the inclusion criteria were enrolled for direct POC-guided lithotripsy using the MB ultraslim endoscope (Fig. 4). The baseline characteristics of the enrolled patients are listed in Table 1.

The technical outcomes of direct POC-guided lithotripsy are listed in Table 2. Direct POC for difficult bile duct stone was successfully performed in all patients either by free-hand insertion (19 patients, 95.0\%) or with intraductal balloon assistance (one patient, 5.0\%). One patient required balloon-assisted technique rather than free-hand insertion for insertion to the distal CBD due to the difficult angle of the orifice of ampulla of Vater.

EHL and LL were attempted in nine (45\%) and 11 (55\%) patients, respectively. Direct POC-guided lithotripsy was successful in 19 (95\%) patients. The one case of failure was due to unsuccessful insertion of the laser probe up to the targeted stone because the less flexible laser probe could not pass through the acute angulated working channel. Complete ductal clearance was achieved in 19 patients (95\%). Ductal clearance was confirmed by direct POC in eight patients (42.1\%). The median time for lithotripsy was 25 minutes (range, 12-45 minutes). Additional MLs were performed in 11 patients (55\%). The median number of ERCP sessions required for complete stone removal was 2 sessions (range, 1-3) (Table 3).

Adverse events occurred in one patient (5\%), who had minor bleeding during EHL but improved with conservative treatment.

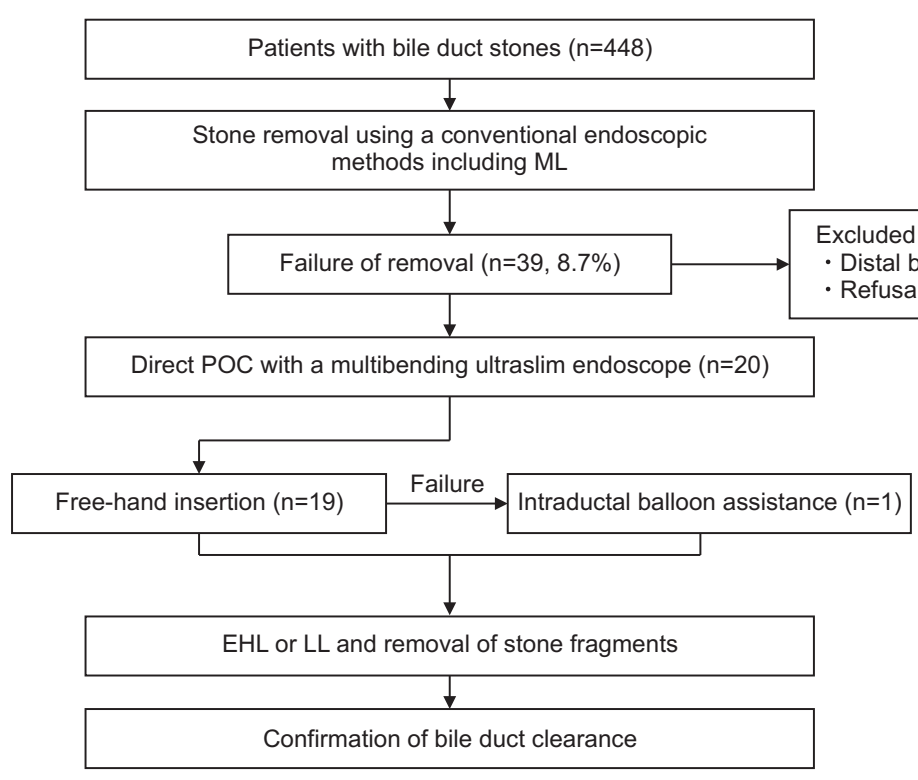

Fig. 4. Patient flowchart through the study.

ML, mechanical lithotripsy; POC, peroral cholangioscopy; EHL, electrohydraulic lithotripsy; LL, laser lithotripsy.

Table 1. Baseline Characteristics of the Patients

\begin{tabular}{lc}
\hline \multicolumn{1}{c}{ Characteristics } & Value (n=20) \\
\hline Age, median (range), yr & $67(32-91)$ \\
Sex, male/female & $6 / 14$ \\
Previous cholecystectomy, No. (\%) & $9(45)$ \\
No. of prior ERCP, mean (range) & $1.5(1.0-4.0)$ \\
Stone size, median (range), mm & $28(10-50)$ \\
No. of stones, mean (range) & $2.0(1.0-5.0)$ \\
Location of stones, No. (\%) & $13(65.0)$ \\
Distal common bile duct & $7(35.0)$ \\
Proximal common bile duct & \\
\hline
\end{tabular}

ERCP, endoscopic retrograde cholangiopancreatography.
Table 2. Technical Outcomes of Direct POC-Guided Lithotripsy for Difficult Bile Duct Stones

\begin{tabular}{lc}
\hline \multicolumn{1}{c}{ Technical outcomes } & Value $(\mathrm{n}=20)$ \\
\hline Success of direct POC, No. (\%) & $20(100)$ \\
Free-hand insertion & $19(95.0)$ \\
Intraductal balloon assistance & $1(5.0)$ \\
Electrohydraulic lithotripsy/laser lithotripsy & $9 / 11$ \\
Success of direct POC-guided lithotripsy, No. (\%) & $19(95.0)^{*}$ \\
Time for lithotripsy, median (IQR), min & $25(12-45)$ \\
Adverse events, No. (\%) & $1(5.0)^{+}$ \\
\hline
\end{tabular}

POC, peroral cholangioscopy; IQR, interquartile range. ${ }^{*}$ One failure of insertion of laser probe up to the targeted stone; ${ }^{\dagger}$ One mild bleeding. 
Table 3. Technical Outcomes of Patients Who Achieved Complete Ductal Clearance after Direct Peroral Cholangioscopy-Guided Lithotripsy

\begin{tabular}{cc}
\hline Technical outcomes & Value $(\mathrm{n}=20)$ \\
\hline Complete ductal clearance, No. (\%) & $19(95.0)$ \\
Use of additional mechanical lithotripsy & $11(55.0)$ \\
ERCP sessions for complete stone removal, & $2(1-3)$ \\
median (range) & \\
\hline
\end{tabular}

ERCP, endoscopic retrograde cholangiopancreatography.

\section{DISCUSSION}

The majority of CBD stones can be extracted by conventional ERCP methods, such as endoscopic sphincterotomy with or without endoscopic papillary large balloon dilatation and ML. The term "difficult bile duct stones" covers a range of difficulties, as well as failure of extraction by conventional ERCP methods. In these cases, additional procedures, such as EHL or LL with direct visualization of choledocholithiasis, are required.

Despite technologic advances, no cholangioscope has been established as the standard instrument. ${ }^{8}$ Clinical application of the dual-operator cholangioscopy system known as the mother-baby scope system is limited by its fragility, technical difficulty, and need for two skilled endoscopists. ${ }^{9}$

Cholangioscopy can also be performed using the SpyGlass DS Direct Visualization System (Boston Scientific), designed for a single operator. This digital single-operator cholangioscopy system is fully disposable and offers improved maneuverability via four-way deflected steering of the scope. It also has higher image quality than the previous version (SpyGlass Legacy). For its application, the approximately $3.5-\mathrm{mm}$-diameter scope is inserted through the accessory channel of the duodenoscope. It is equipped with a $1.2-\mathrm{mm}$ accessory channel and a $1.2-\mathrm{mm}$ suction channel, permitting access by accessories. However, the diameter is insufficient for application of various diagnostic and therapeutic accessories for bile duct lesions. Also, image-enhanced endoscopy cannot be performed using single-operator cholangioscopy. The mother-baby scope system and single-operator cholangioscopy systems cannot be inserted into the biliary tree without assistance. Therefore, a duodenoscope is mandatory for insertion of a cholangioscope.

By contrast, direct POC using an ultraslim endoscope does not require another endoscope or operator. Originally designed for the transnasal approach and pediatric patients, the ultraslim endoscope provides high-quality images, has large working channels, and is cost-effective due to its reusability.

The main obstacle to direct POC using ultraslim endoscopes is the difficulty of inserting the scope into the bile duct because of its relatively acute angle. ${ }^{3}$ Various special accessories, such as a guidewire, overtube, and intraductal balloon have been used to improve the success rate of direct POC using an ultraslim endoscope. ${ }^{10,11}$ However, the procedure is still considered complicated. Loop formation was an issue because the endoscope lacked stiffness; it was not designed specifically for cholangioscopy.

The new MB ultraslim endoscope is more suitable for direct POC. The additional bending section on the distal insertion tube provides unique angulation and improved maneuverability for entering the ampulla of Vater orifice. The $\mathrm{MB}$ endoscope is $23 \mathrm{~cm}$ longer in length $(1,330 \mathrm{~mm})$ than the conventional ultraslim endoscope offering better accessibility within the bile duct. The two working channels have a larger diameter than other platforms, allowing a broader range of accessories to be used. Also, the stiffness of the scope was increased to prevent loop formation.

We evaluated the usefulness of direct POC-guided lithotripsy by using the MB ultraslim endoscope in patients with difficult bile duct stones. The rate of complete ductal clearance of difficult bile duct stones was 95\% (19/20 patients) and adverse events were observed in 5\% (1/20) of the patients using the MB ultraslim endoscope. These findings are in line with a recent meta-analysis, which reported an overall estimated stone clearance rate of $88 \%$ ( $95 \%$ confidence interval, $85 \%$ to $91 \%$ ) in difficult bile duct stone cases using POC-guided lithotripsy, and an overall adverse event rate of $7 \%$ (95\% confidence interval, $6 \%$ to $9 \%)^{12}$

Direct POC using the MB ultraslim endoscope for treatment of difficult bile duct stones also yielded positive technical outcomes, including a high success rate $(100 \%$, $20 / 20$ ). In addition, the free-hand insertion rate was $95 \%$ $(19 / 20)$ and intraductal balloon assistance was needed in only $5 \%(1 / 20)$ of cases. This result represents a significant improvement over the previous studies. A study using the first and second prototype $\mathrm{MB}$ endoscopes in seven patients reported no successful attempts. ${ }^{13}$ Another study that used the third prototype $\mathrm{MB}$ endoscope reported a $72 \%(53 / 74)$ rate of free-hand insertion. ${ }^{14}$ Combined with the high complete ductal clearance rate, the technical outcomes suggest that the new $\mathrm{MB}$ endoscope can overcome the shortcomings of direct POC using previous ultraslim endoscope models. Therefore, it can be considered an efficient approach for treatment of difficult bile duct stones. To our knowledge, no study has evaluated the efficacy of direct POC-guided lithotripsy using the MB ultraslim endoscope.

Despite its advantages, several issues need to be resolved 
for practical application of the MB ultraslim endoscope. It has a $4.9-\mathrm{mm}$ outer diameter at the distal end, which is larger than other dedicated cholangioscopes. Direct POC is recommended for patients with previous sphincteroplasty and a CBD diameter $\geq 8 \mathrm{~mm}$ to ensure safety. Patients with small biliary diameters may not be candidates.

Additionally, the risk of air embolism exists even with minimal use of $\mathrm{CO}_{2}$ throughout the procedure. ${ }^{15,16}$ In our study, when the endoscope was intubated to the distal $\mathrm{CBD}, \mathrm{CO}_{2}$ insufflation was discontinued. Saline irrigation and suction were used to prevent complications such as cholangitis and air embolism. This may have contributed to the low rate of complications.

This study had several limitations. First, it was a singlearm study without a control group, and only 20 patients participated. Small differences in patient outcomes in a study of this size could affect the overall results, so the relevance of the findings to general clinical practice is questionable. Future studies with larger samples and control groups are required to confirm our results. Second, this study was conducted by endoscopists highly experienced in the use of direct POC; only a few practitioners of ERCP have access to direct POC.

In conclusion, direct POC-guided lithotripsy using an MB ultraslim endoscope for the treatment of difficult bile duct stones resulted in a high ductal clearance rate and high technical success rate of intubation of the bile duct, without severe adverse events. It may be a safe and useful endoscopic procedure for patients with difficult bile duct stones. Further development of accessories and standardized protocols will enhance the platform.

\section{CONFLICTS OF INTEREST}

No potential conflict of interest relevant to this article was reported.

\section{ACKNOWLEDGEMENTS}

We thank Olympus Medical Systems for providing the multibending ultraslim endoscope unit. We also thank A Ri Song, RN, Song Ah Jeong, RN, Sun Hwa Cho, RN and all the other nursing staff, for their support and assistance with the procedure. This work was partly supported by the Soonchunhyang University Research Fund.

\section{AUTHOR CONTRIBUTIONS}

Conception and design: J.H.M., Y.N.L. Analysis and interpretation of the data: W.M.L. Drafting of the article: W.M.L. Critical revision of the article for important intellectual content: J.H.M., Y.N.L., I.S.S., J.K.Y., T.H.L., S.W.C., Y.D.C., S.H.P. Final approval of the article: all authors.

\section{ORCID}

Won Myung Lee https://orcid.org/0000-0001-5336-5909 Jong Ho Moon https://orcid.org/0000-0002-3946-9944 Yun Nah Lee https://orcid.org/0000-0001-5588-784X Il Sang Shin https://orcid.org/0000-0003-2199-1942 Tae Hoon Lee https://orcid.org/0000-0002-3545-9183 Jae Kook Yang https://orcid.org/0000-0003-3003-7667 Sang-Woo Cha https://orcid.org/0000-0002-4685-7718 Young Deok Cho https://orcid.org/0000-0002-8089-300X Sang-Heum Park https://orcid.org/0000-0001-6605-1108

\section{SUPPLEMENTARY MATERIALS}

Supplementary materials can be accessed at https://doi. org/10.5009/gnl210355.

\section{REFERENCES}

1. Carr-Locke DL. Therapeutic role of ERCP in the management of suspected common bile duct stones. Gastrointest Endosc 2002;56(6 Suppl):S170-S174.

2. ASGE Standards of Practice Committee, Chathadi KV, Chandrasekhara V, et al. The role of ERCP in benign diseases of the biliary tract. Gastrointest Endosc 2015;81:795803.

3. Moon JH, Choi HJ. The role of direct peroral cholangioscopy using an ultraslim endoscope for biliary lesions: indications, limitations, and complications. Clin Endosc 2013;46:537539.

4. Lee YN, Moon JH, Choi HJ, et al. Direct peroral cholangioscopy using an ultraslim upper endoscope for management of residual stones after mechanical lithotripsy for retained common bile duct stones. Endoscopy 2012;44:819-824.

5. Lee YN, Moon JH, Lee TH, et al. Prospective randomized trial of a new multibending versus conventional ultra-slim endoscope for peroral cholangioscopy without device or endoscope assistance (with video). Gastrointest Endosc 2020;91:92-101.

6. Lee YN, Moon JH, Choi HJ, et al. A newly modified access 
balloon catheter for direct peroral cholangioscopy by using an ultraslim upper endoscope (with videos). Gastrointest Endosc 2016;83:240-247.

7. ASGE Standards of Practice Committee, Chandrasekhara $\mathrm{V}$, Khashab MA, et al. Adverse events associated with ERCP. Gastrointest Endosc 2017;85:32-47.

8. Manes G, Paspatis G, Aabakken L, et al. Endoscopic management of common bile duct stones: European Society of Gastrointestinal Endoscopy (ESGE) guideline. Endoscopy 2019;51:472-491.

9. Nguyen NQ, Binmoeller KF, Shah JN. Cholangioscopy and pancreatoscopy (with videos). Gastrointest Endosc 2009;70:1200-1210.

10. Choi HJ, Moon JH, Ko BM, et al. Overtube-balloon-assisted direct peroral cholangioscopy by using an ultra-slim upper endoscope (with videos). Gastrointest Endosc 2009;69:935940.

11. Larghi A, Waxman I. Endoscopic direct cholangioscopy by using an ultra-slim upper endoscope: a feasibility study. Gastrointest Endosc 2006;63:853-857.
12. Korrapati P, Ciolino J, Wani S, et al. The efficacy of peroral cholangioscopy for difficult bile duct stones and indeterminate strictures: a systematic review and meta-analysis. Endosc Int Open 2016;4:E263-E275.

13. Itoi T, Nageshwar Reddy D, Sofuni A, et al. Clinical evaluation of a prototype multi-bending peroral direct cholangioscope. Dig Endosc 2014;26:100-107.

14. Beyna T, Farnik H, Sarrazin C, Gerges C, Neuhaus H, Albert JG. Direct retrograde cholangioscopy with a new prototype double-bending cholangioscope. Endoscopy 2016;48:929933.

15. Kondo H, Naitoh I, Nakazawa T, et al. Development of fatal systemic gas embolism during direct peroral cholangioscopy under carbon dioxide insufflation. Endoscopy 2016;48 Suppl 1:E215-E216.

16. Efthymiou M, Raftopoulos S, Antonio Chirinos J, May GR. Air embolism complicated by left hemiparesis after direct cholangioscopy with an intraductal balloon anchoring system. Gastrointest Endosc 2012;75:221-223. 